
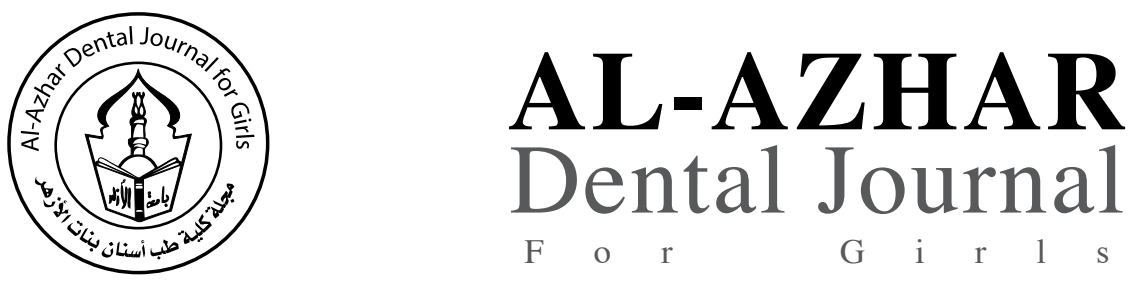

The Official Publication of The Faculty of Dental Medicine For Girls,

Al-Azhar University Cairo, Egypt.

ADJ-for Grils, Vol. 3, No. 3, July (2016) — PP. 201:208

\title{
Evaluation of Nano- Hydroxyapatite versus Hydroxyapatite Crystals in the Treatment of Periodontal Intrabony Defects
}

\author{
Amal Aly Ibrahim EL Said ${ }^{(1)}$, Eatemad Ahmed Shoriebah ${ }^{(2)}$, Naglaa Shawki Essa EL Kilany ${ }^{(3)}$ \\ and Hala Helmi Hazzaa ${ }^{(3)}$
}

Codex : 06/1607

dentaljournal.forgirls@yahoo.com

\begin{abstract}
The aim of the present study was to evaluate clinically and radiographically the effectiveness of nano-crystalline hydroxyapatite ( $\mathrm{NcHA}$ ) versus hydroxyapatite (HA) crystals in the treatment of intrabony periodontal defects. Subjects and Methods: Twelve patients with bilateral defects, with probing depth (PD) $\geq 5 \mathrm{~mm}$ and clinical attachment loss (CAL) $\geq 3 \mathrm{~mm}$ participated in the present study. Subjects were divided randomly into Group 1: 12 sites treated surgically with open flap debridement (OFD) and placement of NcHA (1) as a bone substitute. Group 2: 12 sites treated surgically with open flap debridement and placement of HA (2) as a bone substitute. The following clinical parameters were recorded: $\mathrm{PD}, \mathrm{CAL}$, plaque index $(\mathrm{PI})$, gingival index (GI), alveolar bone level percentage and bone density percentage. Results: At 6 months following therapy, PD and CAL decreased by time, with a statistically significant difference in both groups .On comparison of the mean difference in PD and CAL between the two groups after Mann-Whitney U test was applied at baseline, 3 and 6 months, scores were found to be statistically non-significant $(\mathrm{p}>0.01)$. A significant reduction in PI and GI were recorded following therapy. As regard to radiographic bone measurements, in both groups, alveolar bone level percent decreased by time, with a non-significant difference. The change occurring in the first interval was greater in both groups, with a non-significant difference. In both groups, bone density percent increased by time, with a significant difference. The change occurring in the second interval was greater in both groups, with a non-significant difference between changes occurring in both intervals. Conclusion: Both NcHA and conventional HA led to the improvement of clinical and radiographic parameters over the course of the study. The NcHA group did not show any significant improvement over the HA group.
\end{abstract}

1. Demonstrator in Department of Oral Medicine, Periodontology,Oral Diagnosis and Dental Radiology Faculty of Dental Medicine Al-Azhar University, (Girls Branch)

2. Professor of Oral Medicine, periodontology, Oral Diagnosis and Dental Radiology Department, Faculty of Dental Medicine Al-Azhar University, (Girls Branch)

3. Assistant Professor of Oral Medicine, Periodontology, Oral Diagnosis and Dental Radiology Department,Faculty of Dental Medicine Al- Azhar University, (Girls Branch) 


\section{INTRODUCTION}

The ultimate goal of periodontal treatment is the regeneration of periodontal tissues lost during the disease process. Bone replacement grafts remain among the widely used therapeutic strategies for the correction of periodontal osseous defects ${ }^{(1)}$.

In reviewing the literature, differences in clinical attachment level (CAL) gains varied considerably among the different biomaterials used; because of this heterogenecity in the results among studies, the investigators were unable to draw conclusions about the use of specific graft biomaterials. Until now, autogenous bone is recognized as the gold standard ${ }^{(2)}$.

Nevertheless, autografts increase the morbidity and are limited in availability. These considerations have led to an increased exploration of alternative bone substitute materials. These materials are supposed to be biocompatible, noninfectious, and non-antigenic. Although most are not considered to be osteoinductive, they should at least be osteoconductive ${ }^{(3)}$.

Hydroxyapatite (HA) has been widely applied as bone substitute. It has been the most extensively used substitution materials for artificial bone grafts ${ }^{(4)}$.

A synthetic Nano-crystalline hydroxyapatite (NcHA) bone substitution material has been successfully introduced for the augmentative treatment of space maintaining bone defects after defect fractures and cystectomies without revealing negative side effects ${ }^{(5)}$.

Although this NcHA material is different from microcrystalline bone substitution materials regarding solubility, its chemical composition corresponds to that of bone mineral. The particle size was reported to be $18 \mathrm{~nm}$ in average, which allows for an accelerated substitution by vital bone ${ }^{(6)}$.

Preliminary experimental studies have shown that Nano sized ceramics may represent a promising class of bone graft substitutes due to their improved osseointegrative properties ${ }^{(7)}$.

Moreover NcHA has been used in animal study in adult domestic pigs for observation of the regenerative potential in bony defects histologically; there was suitable osseointegration and osteoconduction of the used material. Complete resorption of the nanoparticle hydroxyapatite had taken place after 12 weeks. It can be concluded that the evaluated nanoparticle hydroxyapatite met the clinical requirements for a bone substitute material within the limits of this experimental setting ${ }^{(8)}$.

\section{SUBJECTS AND METHODS}

Twelve patients with 24 intrabony defects and age range of 40-55 years, selected consecutively between November 2013 and August 2014 from those referred to the Department of oral medicine, periodontology, diagnosis and radiology, Faculty of Dentistry Al-Azhar University seeking for periodontal treatment.

The nature of this investigation was explained in detail, and the patients signed an informed consent form.

\section{Inclusion Criteria}

1. The patients were free from any systemic diseases as evaluated by modified Cornell medical index ${ }^{(9)}$ and did not receive any antibiotic therapy during the last 3 months prior to treatment or periodontal therapy.

2. Presence of at least one pair of matched two or three-wall periodontal intrabony defect with a $P D \geq 5 \mathrm{~mm}$ and attachment loss $\geq 3 \mathrm{~mm}$, four weeks following the initial periodontal therapy on the basis of having intrabony defect(s) and radiographic bone loss as detected by periapical radiographs.

3. All patients were willing and able to return for multiple follow up visits in a 3 and 6-month clinical trial.

4. Good oral hygiene.

\section{Exclusion Criteria:}

1- Patients with teeth exhibiting furcation involvement, inadequate endodontic treatment, or overhanging margins.

2- Pregnancy or lactation.

3- Smoking or formal smoking. 
The patients selected on the above criteria were then explained about the treatment procedure and there written consent was optained. Four weeks following phase I therapy a periodontal re-evaluation was perfomed to confirm the suitability of the sites for the study.

The following clinical parameters were recorded at baseline 3 and 6 months postoperatively:

- Pocket depth(PD) The distance from the base of the pocket to the gingival margin

- Clinical attachment level (CAL) The distance from the base of the periodontal pocket to cement-enamel junction

- Plaque index (PI).

- Gingival index(GI)

Radiographic assessment-Standardized reproducible periapical radiographs for each treated site were evaluated preoperatively, three and six months after therapy.

\section{Surgical procedure ${ }^{(10)}$.}

The surgical procedures were performed using routine local anesthetics (2\%Xylocaine with epinephrine $1: 100,000$ )

- Reflection of full thickness flaps was utilized to retain as much as soft tissue as possible. The flaps were raised on the buccal and lingual/palatal aspects of teeth, including at least one tooth distal to the area being treated.

- The inner surface of the flap was carefully curetted to remove dento-gingival epithelium and granulation tissue, and then complete debridement and root planning were accomplished with hand instruments. The surgical area was rinsed with copious amount of sterile saline.

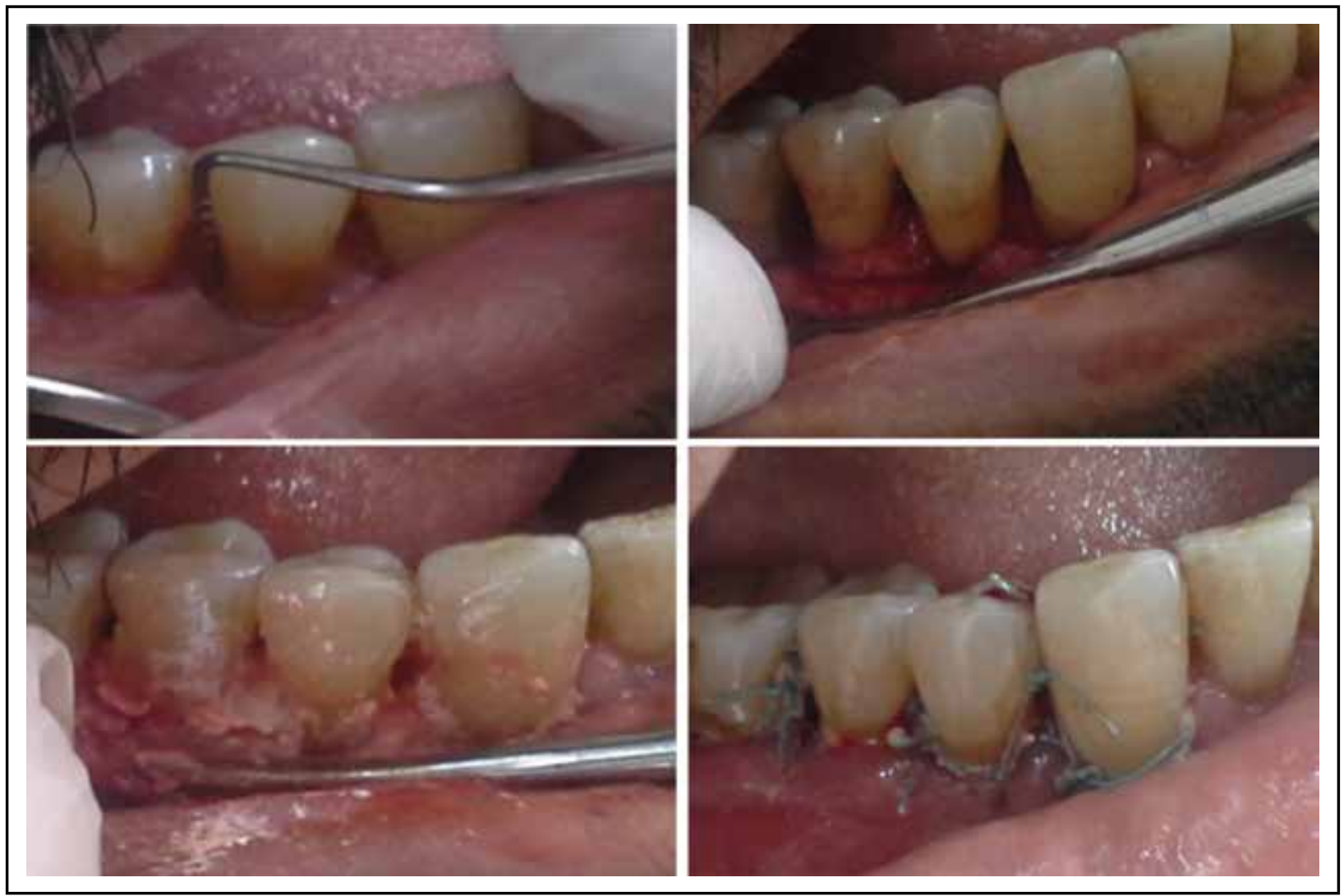

Fig. (1) : Group (1) (a)preoperative probing depth distal to lower right first premolar using Williams graduated probe.(b) elevation of mucoperiosteal flap.(c) application of nanohydroxyapatite in the intrabony defect.(d) the flap tissue is secured with resorbable suture. 

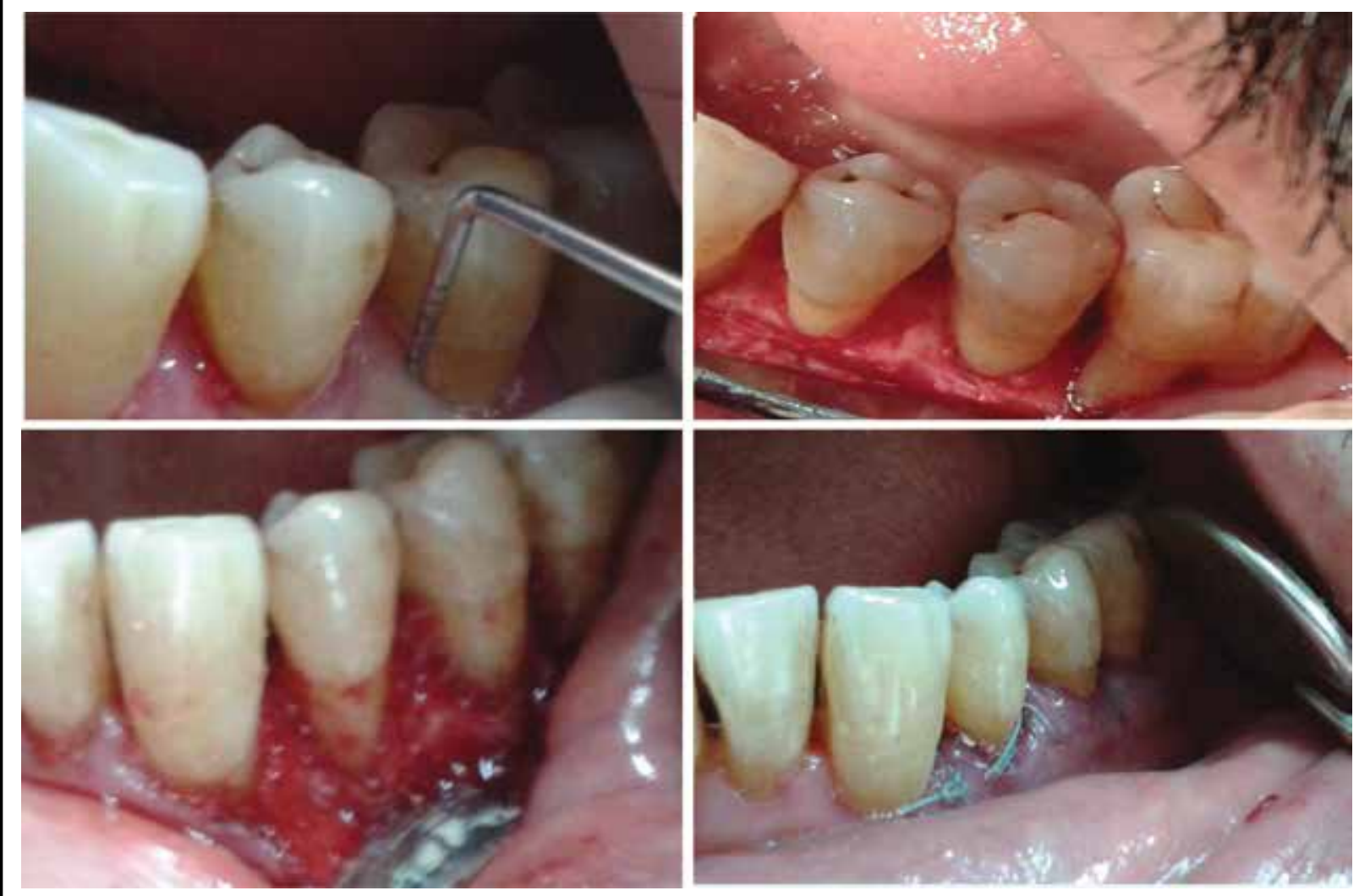

Fig. (2) Group (2)(a)preoperative probing depth mesial to lower left second premolar using Williams graduated probe.(b) elevation of mucoperiosteal flap.(c)application of nanohydroxyapatite in the intrabony defect.(d) the flap tissue is secured with resorbable suture.

- For group $1 \mathrm{NcHA}$ bone substitute was mixed with saline, and applied into the defects.

- For group 2 HA bone substitute was mixed with saline, and applied into the defects.

- Flaps were coronally repositioned in place with interrupted suture using primary soft tissue closure utilizing 4.0 black silk sutures.

\section{Statistical Analysis}

Clinical data and radiographic data were presented as mean and standard deviation (SD) values. Data were explored for normality using Kolmogorov-Smirnov test of normality. The results of Kolmogorov-Smirnov test indicated that most of data were not normally distributed (non-parametric data) so non-parametric tests were used for the comparisons.
Kruskal-Wallis test was used to compare between the three observation times. MannWhitney U test was used for comparisons between changes occurring in each interval, as well as for comparison between both groups nano-crystalline Hydroxyapatite (NcHA) and Hydroxyapatite (HA). The significance level was set at $p \leq 0.05$. Statistical analysis was performed with SPSS 16.0 (Statistical Package for Scientific Studies, SPSS, Inc., Chicago, IL, USA) for Windows.

\section{RESULTS}

During the course of this study, no adverse tissue reaction, infections or delayed wound healing were reported in all sites of all groups.

- Probing depth Comparing both groups, revealed a significantly lower probing depth at 6 months and a greater change in the $2^{\text {nd }}$ interval in NcHA group (Fig. 3). 
- Clinical attachement loss (CAL) Comparing both groups, revealed a significantly lower Clinical attachement loss at 6 months and a greater change in the 1 st interval in $\mathrm{NcHA}$ (Fig. 4).

- Plaque index: Comparing both groups, revealed a greater change in $\mathrm{NcHA}$, with a nonsignificant difference in all evaluation times and non- significant difference in change in both intervals (Fig. 5).

Gingival index: Comparing both groups, revealing greater change in $\mathrm{NcHA}$ in the 2nd interval,

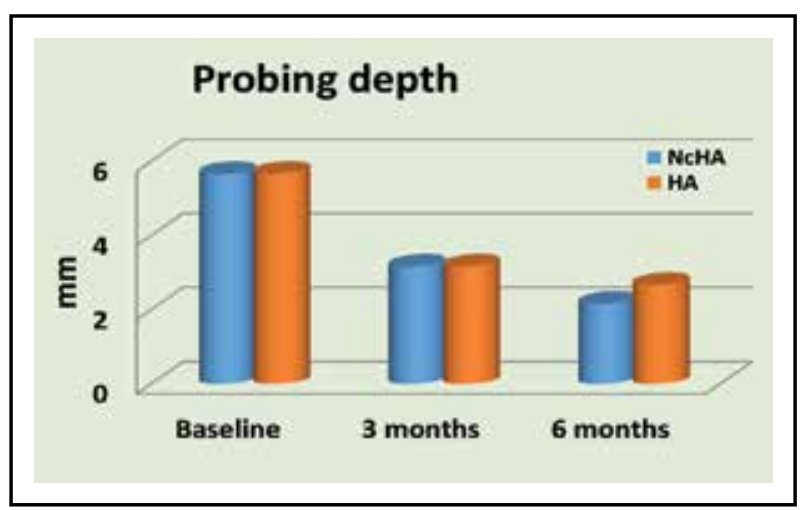

Fig. (3)The mean probing depth at base line, 3 months and 6 months evaluation in both study groups

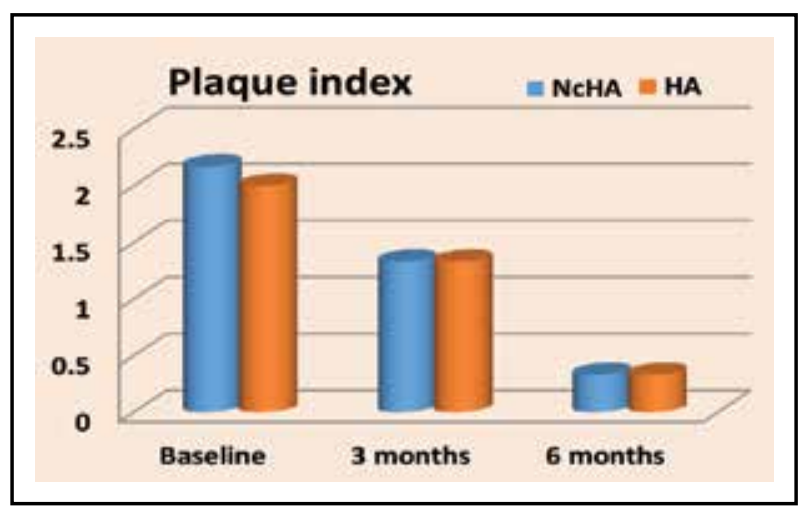

Fig. (5) The mean Plaque index at base line, 3 months and 6 months in both groups. with a non-significant difference in all evaluation times and non- significant difference in change in both intervals (Fig. 6).

- Alveolar bone level percentage: Comparing both groups, revealed a non-significant difference in all evaluation times and non- significant difference in change in both intervals (Fig. 7).

- Bone density percentage: Comparing both groups, revealed a significant difference in all evaluation times, except at 6 months. Moreover, there was a significant difference in change in both intervals, and throughout the study (Fig. 8).

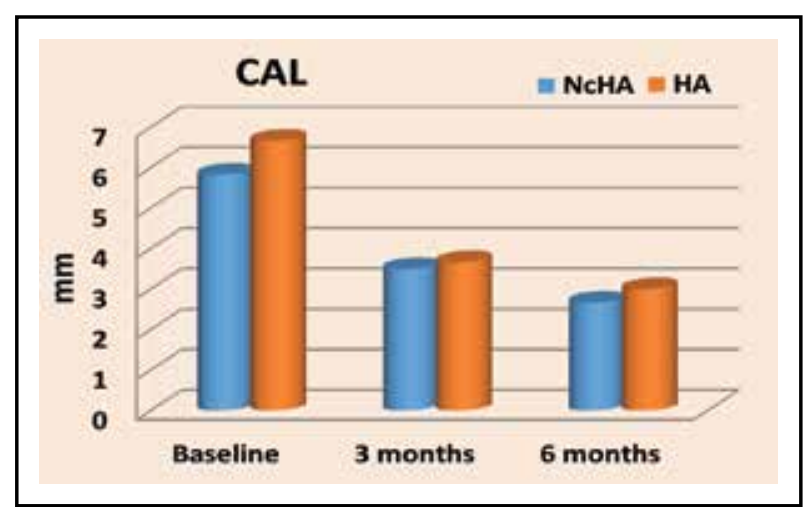

Fig. (4) The mean Clinical attachment loss at base line, 3 months and 6 months evaluation in both groups.

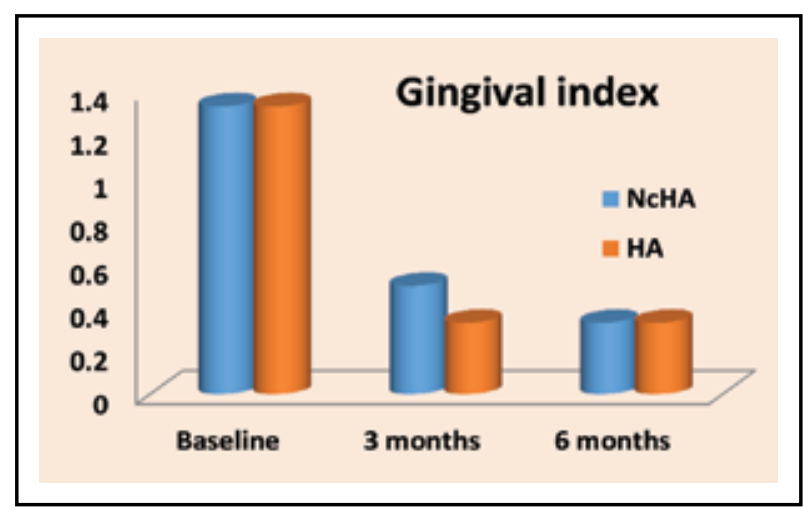

Fig. (6) The mean gingival index at base line, 3 months and 6 months in both groups. 


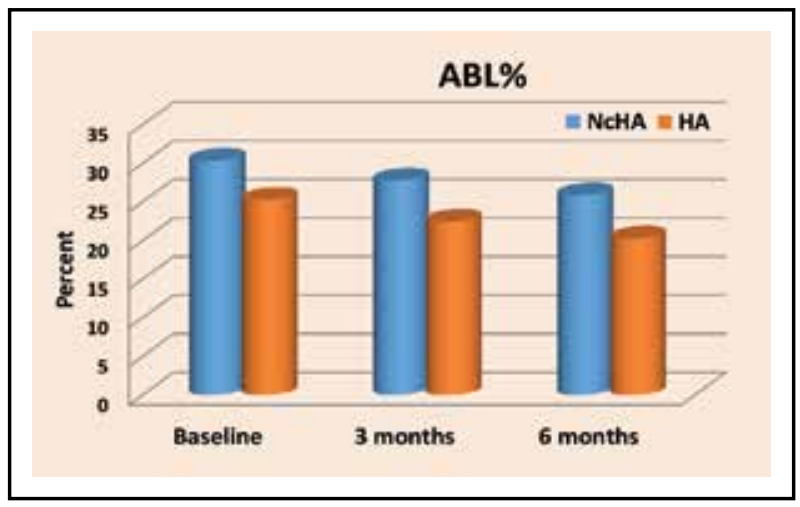

Fig. (7) The mean alveolar bone level percentage ABL\% at base line, 3 months and 6 months in bothgroups.

\section{DISCUSSION}

Concerning the results of the present study, an improvement in both clinical and radiographic parameters was shown with the use of conventional HA graft which was found to be statistically significant; with a continued significant reduction from 3 to 6 months. In accordance with the observations of the current study, ${ }^{(11)}$ Bansal demonstrate a decrease in PD from baseline to 3 months and 6 months. Similar observations were also seen concerning the relative attachment level. These findings signify the use of HA graft in the clinical resolution of intrabony defects.

The improvement in the clinical probing depth and relative attachment level was well supported by the decrease in the radiographic area of the intrabony defect in the HA group of the present study, which was determined in a manner similar to that as described by Eickholz et al. (2004). The observations of the present study show a decrease in the size of the defect from baseline to 3 and 6 months respectively. Also, the difference from 3 months to 6 months was statistically non-significant.

In accordance with the radiological observations of the current study, Scabbia who compare between hydroxyapatite/collagen/chondroitin sulphate biomaterial and a bovine derived hydroxyapatite xenograft $\left(\right.$ Bio-Oss $\left.^{\circledR}\right)$ in the treatment of deep intra-osse-

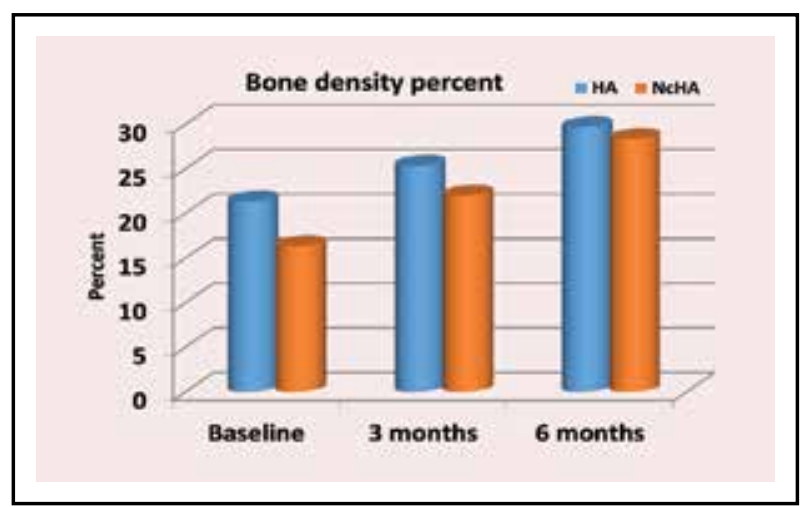

Fig. (8) The mean bone density percentage at base line, 3 months and 6 months in both groups.

ous defect, ${ }^{(13)}$ and Okuda who use Platelet-rich plasma combined with a porous hydroxyapatite graft for the treatment of intrabony periodontal defects in humans and evaluate the depth of the intrabony defect radiographically and reported a significant gain in the bone height and reduction of the depth of the defect $^{(14)}$.

Regarding the percentage of change in bone density, there was statistically significant increase in bone density at 3 and 6 months with the use of HA. Signifies that use of HA graft results in resolution of the intrabony defect. However, the nature of the restoration of the defect that whether the graft acted as a filler material or allowed for ingrowth of the bone cannot be inferred from the clinical and radiographic observations of the present study. These radiographic changes are in accordance with previous studies showing similar results ${ }^{(14,15,16)}$.

In the NcHA group, there is reduction of PD. In a study by Kasaj that evaluated the clinical efficacy of NcHA paste in intrabony defects and reported PD reduction and CAL gain which was slightly higher than the present study ${ }^{(17)}$. The slightly higher PD reduction and CAL gain may be due to differences in the physicochemical and structural characteristics between the two graft types, which might lead to differences in the regenerative and osteoconductive properties ${ }^{(17)}$. 
The use of NcHA in the treatment of periodontal defects was evaluated in the pioneer study of Zuev who found that NcHA paste to be not inferior to bone transplants and it was particularly effective in the treatment of periodontitis at the stage of abscesses $^{(18)}$. Kasaj ${ }^{(17)}$ stated that at 6 months after surgery, the treatment of intrabony periodontal defects with an NcHA paste produced clinically and statistically significant PD reductions and CAL gains compared to open flap debridement alone.

Furthermore, Heinz ${ }^{(19)}$ compared clinical outcomes of papilla preservation flap surgery with or without the application of NcHA bone graft substitute. They observed that after 6 months statistically significant reduction in probing pocket depths and gain in probing bone levels in the test group. These observations were in accordance with that of the current study.

The current study also demonstrates a statistically significant reduction in area of defect and increase in radiodensity of the defect from baseline to 6 months and from 3 to 6 months with the use of $\mathrm{NcHA}$. However, the decrease in area from baseline to 3 months was statistically insignificant. This might be because of the very small particle size and early resorption within 12 weeks as reported by Thorwarth ${ }^{(20)}$ who used microradiography to assess the mineralization content and degradation of the test material; Bansal ${ }^{(1)}$ also reported similar findings.

Regarding the percentage of change in bone density, there was statistically significant increase in bone density at 3 and 6 months with the use of $\mathrm{NcHA}$. The significant change suggests that $\mathrm{NcHA}$ may be more advantageous in treating intrabony defects. In accordance, Schnettler ${ }^{(21)}$ found that NcHA binds to the bone and stimulates bone healing by stimulation of osteoblast activity.

Another clinical study that used the split mouth technique to investigate the effect of extraction socket augmentation with a synthetic NcHA showed that the degree of gingival invagination and probing depth were significantly reduced on augmented extraction site ${ }^{(22)}$.

The reduction in mean plaque and gingival indices scores within the study groups from baseline to 3 , and 6 months was statistically significant.owever, there was no significant difference between the two groups regarding the reduction of scores for the same intervals of the study. These findings were consistent with previous studies ${ }^{(23,24,2526)}$ which have shown statistically significant changes in plaque and gingival indices within the groups from baseline but no statistically significant differences were observed between the two groups. The improved gingival and plaque index scores in both groups showed the increased level of oral health awareness among patients and a good maintenance of oral hygiene by them throughout the study period.

\section{REFERENCES}

1. Papapanou PN, Tonetti MS : Diagnosis and epidemiology of periodontal osseous lesions. Periodontol (2000); 22:8-21.

2. Trombelli L, Heitz-Mayfield LJ, Needleman I, Moles D, Scabbia A: A systematic review of graft materials and biological agents for periodontal intraosseous defects. J Clin Periodontol. (2002);29 Suppl 3:117-35.

3. Khan SN, Cammisa FP Jr, Sandhu HS, Diwan AD, Girardi FP, Lane JM: The biology of bone grafting. J Am Acad Orthop Surg.(2005);13:77-86.

4. Frieb, J Warner, F Schuth, KSW Sing, J Weitkamp Weitkamp Eds.Hand book of Porous solids Wiley-VCH, 2002,2923pp.

5. Bezrukov VM, Grigor'iants LA, Zuev VP, Pankratov AS: [The surgical treatment of jaw cysts using hydroxyapatite with an ultrahigh degree of dispersity]. Stomatologiia (Mosk). (1998);77:31-5.

6. Strietzel FP, Reichart PA, Graf HL: Lateral alveolar ridge augmentation using a synthetic nano-crystalline hydroxyapatite bone substitution material (Ostim): preliminary clinical and histological results. Clin Oral Implants Res(. 2007);18:743-51.

7. Chris Arts, J.J., Verdonschot, N., Schreurs, B.W. \& Buma,P: The use of a bioresorbable nano-crystalline hydroxyapatite paste in acetabular bone impaction grafting. Biomaterials (2006) 27, 1110-1118. 
8. Huber FX, McArthur N, Heimann L, Dingeldein E, Cavey H, Palazzi X, Clermont G, Boutrand JP: Evaluation of a novel nanocrystalline hydroxyapatite paste Ostim in comparison to Alpha-BSM - more bone ingrowth inside the implanted material with Ostim compared to Alpha BSM-BMC Musculoscelet Disord (2009) Dec 22;10:164.

9. Kerr DA, Millard HD: Oral diagnosis. $1965 ; 2^{\text {nd }}$ edition: 17.

10. Chitsazi MT,Shirmohammadi A, Faramarzie M, Pourabbas $\mathrm{R}$, Rostamzadeh A:A clinical comparison of nanocrystalline hydroxyapatite(Ostim) and autogenous bone graft in the treatment of periodontal intrabony defects. Med Oral Patol Oral Cir Bucal. 2011;16 (3)e448-53.

11. Bansal R, Patil S, Chaubey K K, Thakur R K, Goyel P: Clinical evaluation of hydroxyapatite and $\beta$-tricalcium phosphate composite graft in the treatment of intrabony periodontal defect: A clinico-radiographic study.J Indian Soc Periodontol. 2014 Sep-Oct; 18(5): 610-617.

12. Eickholz P, Horr T, Klein F, Hassfeld, Kin TS. Radiographic parameters for prognosis of periodontal healing of infrabony defects: Two different definitions of defect depth. J Periodontol. 2004;75:399-407.

13. Scabbia A, Trombelli L. A comparative study on the use of hydroxyapatite/collagen/chondroitin sulphate biomaterial and a bovine derived hydroxyapatite xenograft (BioOss $\left.{ }^{\circledR}\right)$ in the treatment of deep intra-osseous defect. J Clin Periodontol. 2004;31:348-55.

14. Okuda K, Tai H, Tanabe K, Suzuki H, Sato T, Kawase $\mathrm{T}$, et al,Platelet-rich plasma combined with a porous hydroxyapatite graft for the treatment of intrabony periodontal defects in humans: a comparative controlled clinical study. J Periodontol. 2005; 76:890-8.

15. Ouyang XY and Qiao J. Effect of platelet-rich plasma in the treatment of periodontal intrabony defects in humans. Chin Med J (Engl). 2006; 119:1511-21.

16. Shirakata Y, Setoguchi T, Machigashira M, Matsuyama T, Furuichi Y, Hasegawa K et al. Comparison of injectable calcium phosphate bone cement grafting and open flap debridement in periodontal intrabony defects: a randomized clinical trial. J Periodontol. 2008, 79(1):25-32.

17. Kasaj A, Rohrig B, Zafiropoulos GG, Willershausen B. Clinical evaluation of nanocrystalline hydroxyapatite paste in the treatment of human periodontal bony defects-A randomized controlled clinical trial: 6 month results. J Periodontol. 2008;79:394-400.

18. Zuev VP, Dmitrieva LA, Pankratov AS, Filatova NA. The comparative characteristics of stimulators of reparative osteogenesis in the treatment of periodontal diseases. Stomatologia. 1996;75:31-4.

19. Heinz B, Kasaj A, Teich M, Jepsen S. Clinical effects of nanocrystalline hydroxyapatite paste in the treatment of intrabony periodontal defects: A randomized controlled clinical study. Clin Oral Investig. 2010;14:525-31.

20. Thorwarth, M., Schultze-Mosgau, S., Kessler, P., Wiltfang, J. \& Schlegel, K: A Bone regeneration in osseous defects using a resorbable nanoparticular hydroxyapatite. Journal of Oral and Maxillofacial Surgery. (2005) 63, 1626-1633.

21. Schnettler R, Stahl JP, Alt V, Pavlidis T, Dingeldein E, Wenisch S. Calcium phosphate - based bone substitute. Eur J Trauma 2004;4:219-29.

22. Reichert C, Matthias Wenghoefer, Eric Kutschera, Werner Gotz, Andereas Jager: Ridge preservation with synthetic NcHA reduces the severity of gingival invagination - a prospective clinical study. J Orofac Ortho 2014;74:7-15.

23. Dori F, Kovacs V, Arweiler NB, Huszar T, Gera I, Nikolidakis D et al, Effect of platelet-rich plasma on the healing of intrabony defects treated with an anorganic bovine bone mineral: a pilot study. J Periodontol. 2009; 80:1599-605.

24. Pradeep AR, Pai S, Garg G, Devi P and Shetty SK. A randomized clinical trial of autologous platelet-rich plasma in the treatment of mandibular degree II furcation defects. J Clin Periodontol. 2009; 36:581-8.

25. Yilmaz S, Cakar G, Kuru B, Dirikan S and Yildirim B. Platelet-rich plasma in combination with bovine derived xenograft in the treatment of deep intrabony periodontal defects: a report of 20 consecutively treated patients. Platelets. 2009; 20:432-40.

26. Kaushick BT, Jayakumar ND, Padmalatha $\mathrm{O}$ and Varghese S. Treatment of human periodontal infrabony defects with hydroxyapatite + beta tricalcium phosphate bone graft alone and in combination with platelet rich plasma: a randomized clinical trial. Indian J Dent Res. 2011; 22:505-10. 